Pacific Journal of Mathematics

THE THEORY OF ALMOST PERIODIC FUNCTIONS IN 


\title{
THE THEORY OF ALMOST PERIODIC FUNCTIONS IN CONSTRUCTIVE MATHEMATICS
}

\author{
JAMES BROM
}

\begin{abstract}
In this paper we develop a constructive theory of continuous almost periodic functions. We expose those aspects of the standard theory that are not constructive and give constructive substitutes. For example, it is not true in constructive mathematics that each trigonometric polynomial is almost periodic. A trigonometric polynomial is almost periodic if and only if its exponents are rationally discrete. We obtain a constructive proof of Bohr's fundamental theorem that leads to a computational method for uniform approximations to continuous almost periodic functions by trigonometric polynomials.
\end{abstract}

In the following it is desirable that the reader is familiar with the principles of constructive mathematics which are presented in [2].

Introduction. The notion of an almost periodic function was introduced by the mathematician Harald Bohr [4] in 1924. The notion is a generalization of purely periodic functions.

Much of the standard theory of almost periodic functions is not valid constructively. That is, in many cases the numbers that are asserted to exist are not computable. Nevertheless, Bohr's basic definitions form the basis for the development of a constructive theory.

Definitions. A continuous function $f: \mathbf{R} \rightarrow \mathbf{C}$ is almost periodic if there is $L: \mathbf{R}^{+} \rightarrow \mathbf{R}^{+}$such that in every interval of length $L(\epsilon)$ there is an $\epsilon$-translation number $t$; that is, a number $t$ such that

$$
|f(x+t)-f(x)|<\epsilon . \quad(x \in \mathbf{R})
$$

We call $L$ a modulus of almost periodicity for $f$.

Almost periodic functions occur frequently. For example, note that $f(x)=\sin \sqrt{2} x+\sin x$, a sum of two purely periodic functions, is almost periodic.

In the classical theory of almost periodic functions, they are bounded and uniformly continuous; the uniform limit of a sequence of almost periodic functions is itself almost periodic; and the class of almost periodic functions is linear and closed under the operations of multiplication, conjugation, translation and the operation of taking the modulus. Some of these properties fail constructively. Others are 
valid from the constructive point of view but by reasons different from those given in the classical theory.

In the constructive theory, we assert that a continuous almost periodic function $f$ is bounded. But boundedness is not enough in constructive mathematics to claim that the supremum of $f$, denoted by $\|f\|_{x}$, exists. Nevertheless, it follows from almost periodicity that we can compute $\|f\|_{\infty} ;$ in fact,

$$
\|f\|_{\infty}=\lim _{n \rightarrow \infty}\left\{\sup _{x \in[-n, n]}|f(x)|\right\}
$$

A proof of this appears in [6]. The classical argument that an almost periodic function $f$ is uniformly continuous in the sense that there is $\omega: \mathbf{R}^{+} \rightarrow \mathbf{R}^{+}$such that $|f(x)-f(y)|<\epsilon$ whenever $x, y \in \mathbf{R}$ and $|x-y|<$ $\omega(\epsilon)$ is valid constructively. Also, the classical arguments which show that a uniform limit of a sequence of almost periodic functions is almost periodic and that the class of almost periodic functions is closed under conjugation, translation, and the operation of taking the modulus are valid constructively. However, unlike the classical theory, the moduli of almost periodicity of two almost periodic functions is not always enough to construct either the modulus of almost periodicity for their sum of the modulus of almost periodicity for their product. In fact, a constructive proof of either the closure under addition or closure under multiplication properties would entail a constructive proof of the statement that $\alpha$ is either equal to zero or different from zero whenever $\alpha$ is a non-negative real number. (To see this note that $e^{i \alpha x}$ is almost periodic if and only if $\alpha=0$ or $\alpha \neq 0$. For a proof see either [5] or [6]. For $\alpha \geqq 0$, consider $e^{i \alpha x}$ which is the product of the almost periodic functions $e^{i(1+\alpha) x}$ and $e^{-i x}$. If the closure under multiplication property were valid, then we would be able to conclude that either $\alpha=0$ or $\alpha>0$. If the closure under addition property is valid and if $\alpha \geqq 0$ then $e^{i(1+\alpha) x}-e^{i x}$ is almost periodic; that is, $e^{i x}\left(e^{i \alpha x}-1\right)$ is almost periodic. But then $\left|e^{i \alpha x}-1\right|$ is almost periodic and $\left\|e^{i \alpha x}-1\right\|_{x}>0$ implies $\alpha>0$ and $\left\|e^{i \alpha x}-1\right\|_{\infty}<1$ implies $\alpha=0$.)

Since it is not true in constructive mathematics that, in general, a sum of almost periodic functions is almost periodic; it is an interesting question to ask when a trigonometric polynomial is almost periodic.

THEOREM. Suppose $c_{1}, c_{2}, \cdots, c_{n} \in \mathbf{C}$ such that $\left|c_{k}\right|>0$ $(k=1,2, \cdots, n)$. Then the trigonometric polynomial $P(x)$ defined by

$$
P(x) \equiv \sum_{k=1}^{n} c_{k} e^{i \lambda_{k} x} \quad(x \in \mathbf{R})
$$

is almost periodic if and only if $\lambda_{1}, \lambda_{2}, \cdots, \lambda_{n}$ are rationally discrete. 
Definition. $\quad \lambda_{1}, \lambda_{2}, \cdots, \lambda_{n} \in \mathbf{R}$ are rationally discrete if $\sum_{k=1}^{n} j_{k} \lambda_{k}=0$ or $\sum_{k=1}^{n} j_{k} \lambda_{k} \neq 0$ whenever $j_{1}, j_{2}, \cdots, j_{n}$ are integers.

An elegant proof of this theorem that employs a constructive variant of a theorem originally due to Kronecker appears in [6, pp. 359-361]. For a different constructive proof that is based on Fejér kernels, see [5, pp. 5-14].

The notion of the mean value of a function plays a key role in the development of the classical theory of almost periodic functions. Constructively, we can compute the mean value of an almost periodic function $f$. (See [1, pp. 12-14].) If we denote the mean value by $M_{t}\{f(t)\}$, then we have

$$
M\{f(t)\}=\lim _{T \rightarrow \infty} \frac{1}{2 T} \int_{-T}^{T} f(t) d t=\lim _{T \rightarrow \infty} \frac{1}{T} \int_{0}^{T} f(t) d t .
$$

The fundamental result in Bohr's work is that each almost periodic function, like each purely periodic function, can be represented by its Fourier series

$$
f(x) \sim \sum_{k=1}^{\infty} c_{k} e^{i \lambda_{k} x},
$$

where $M_{x}\left\{|f(x)|^{2}\right\}=\sum_{k=1}^{x}\left|c_{k}\right|^{2}$. Using the notion of mean value, a classical mathematician could define the inner product space of almost periodic functions such that $\|f\|^{2}=M_{x}\left\{|f(x)|^{2}\right\}$. Then Bohr's result could be interpreted to say that the series converges to $f$ in the mean; that is, in the \|\| -sense.

Once Bohr established his fundamental theorem, he was able to show that any continuous almost periodic function is the limit of a uniformly convergent sequence of trigonometric polynomials. This is the main result of his second paper. In view of the fact that the converse of this result was also true, the classical theory contained a characterization of almost periodic functions.

Constructively, we do not assert that all trigonometric polynomials are almost periodic. So we cannot assert that the class of functions that can be uniformly approximated by trigonometric polynomials is the same as the class of almost periodic functions. However, it is the purpose of this paper to show that Bohr's fundamental result has a constructive version and that, not only is his second result valid, the second result follows from the first. Once this is established, we can conclude that the class of almost periodic functions is a proper subset of the uniform closure of the trigonometric polynomials.

REMARK. A constructively weaker notion than almost periodicity, call it weak almost periodicity, is desired in order to describe all those 
functions that are uniform limits of sequences of trigonometric polynomials. It is an unsolved problem to determine what the notion of weak almost periodicity should be.

\section{Fundamental theorem of almost periodic}

functions. Bohr's nonconstructive methods for establishing the fundamental result of the theory are based on reducing the problem to a problem of purely periodic functions. (See [4].) Weyl [8] in 1927 and Rellich [7] in 1935 based their proofs on properties of compact Hermitian operators. These methods, although shorter and less complicated than Bohr's, lost the elementary flavor of Bohr's methods. Nevertheless, a constructive version of the functional analysis approach is possible.

REMARK. I have been unsuccessful in any attempt to compute the trigonometric series directly from the properties of purely periodic functions.

We wish to construct coefficients $c_{1}, c_{2}, c_{3}, \cdots$ and frequencies $\lambda_{1}, \lambda_{2}, \lambda_{3}, \cdots$ for which we can write

$$
f \sim \sum_{k=1}^{\infty} c_{k} e^{i \lambda_{k} x} \quad(x \in \mathbf{R})
$$

where the series converges to $f$ in the mean sense.

The method by which we shall establish 1.1 is based on the idea that the exponentials in the expansion of $f$ should correspond to eigenvectors of the convolution operator $g \rightarrow f * g$ [1, pp. 67-74], where

$$
f * g(x) \equiv \underset{t}{M}\{f(x-t) g(t)\}
$$

So we begin by constructing an inner product space $H$, say, such that the following properties are valid:

(i) $H$ contains $f$ and its translates.

(ii) $H$ is closed under the convolution operation $*$.

(iii) $(g, h) \equiv M_{t}\{g(t) \overline{h(t)}\}$ for all $g, h \in H$.

(iv) Uniform limits of sequences of almost periodic functions in $H$ are in $H$.

REMARK. If two continuous functions $g$ and $h$ are jointly almost periodic; that is, if there is $L: \mathbf{R}^{+} \rightarrow \mathbf{R}^{+}$such that in every interval of length $L(\epsilon)$ there is an $\epsilon$-translation number $t$ that works for both $g$ and $h$; then their sum, product, and convolution product are almost periodic. Classically, any finite collection of almost periodic functions is jointly almost periodic; in which case, $H$ can be taken to be the entire 
class of almost periodic functions. Unfortunately, in constructive mathematics, it is not true that a finite collection of almost periodic functions is jointly almost periodic. (If $\alpha \geqq 0$ then joint almost periodicity of $e^{i(1+\alpha) x}$ and $e^{-i x}$ would imply that $e^{i \alpha x}$ is almost periodic; that is, either $\alpha=0$ or $\alpha \neq 0$.) So the situation in constructive mathematics is that we must be very careful in our construction of $H$ if we are to have an inner product space with the above properties.

It is shown in [5, pp. 71-76] that the construction of $H$ is achieved without much trouble once it is recognized that translates of an almost periodic $f$ are jointly almost periodic, so that linear combinations of translates of $f$ are almost periodic; and that a convolution product between two such linear combinations is the uniform limit of a sequence of linear combinations of translates of $f$.

In order to take advantage of the constructive spectral properties of a compact, Hermitian operator we reduce the problem, as in the classical theory, to the case the convolution operator $g \rightarrow f * g$ is Hermitian by noting that $f=f_{1}+i f_{2}$, where $f_{1}(x) \equiv(f(x)-\overline{f(-x)}) / 2$ and $f_{2}(x) \equiv$ $(f(x)-f(-x)) / 2 i$, and that the convolution operators $g \rightarrow f_{1} * g$, $g \rightarrow f_{2} * g, g \rightarrow f_{2} * g$ are Hermitian (since $f_{k}(x)=\overline{f_{k}(-x)}, k=1,2$ ).

REMARK. In order that $f_{1}, f_{2}$ are in $H$ we construct $H$ such that it is closed under the operation of taking the involution, denoted by \# . (See [5, pp. 71-76].)

Definition. The involution of a function $g$ is $g^{\#}$ where $g^{\#}(x) \equiv$ $\overline{g(-x)}$ for each $x \in \mathbf{R}$.

Now that we can assume the convolution operator is Hermitian, what about compactness?

The convolution operator $A: H \rightarrow H$, defined by the equation

$$
A h \equiv f * h, \quad(h \in H)
$$

is compact in classical mathematics: In Weyl's proof [8] the existence of the trigonometric expansion of $f$ follows from the spectral theory of compact, Hermitian operators. A crucial step is showing that the finite dimensional $A$-invariant spaces in the spectral decomposition are invariant under all translation operators. Consequently, by means of a classical theorem concerning families of unitary operators on finite dimensional spaces, it is established that each of the $A$-invariant, finite dimensional spaces has a basis consisting of exponential functions. Then it is easy to show that these exponentials are precisely the exponentials in the expansion of $f$.

The constructive arguments proceed along the same lines in spite of the fact that the classical theory of the spectral properties of compact 
operators does not have a computational flavor and the fact that the theorem on unitary transformations of a finite dimensional space is non-constructive.

We shall analyze the spectral properties of the convolution operator from the point of view that this operator is approximately finite dimensional; that is, it is the limit in the operator norm sense of a sequence of finite dimenisonal operators.

Definition. An operator $T$ on a Banach space $X$ is finite dimensional if it has the form

$$
T x=\sum_{k=1}^{n}\left\langle x, U_{k}\right\rangle x_{k}
$$

where $n$ is a positive integer; $U_{1}, U_{2}, \cdots, U_{n}$ are bounded linear functionals on $X$ with values $\left\langle x, U_{k}\right\rangle$ for each $x \in X(k=1,2, \cdots, n)$; and $x_{1}, x_{2}, \cdots, x_{n}$ are linearly independent vectors in $X$.

Definition. We say vectors $x_{1}, x_{2}, \cdots, x_{n}$ are linearly independent in the Banach space $(X,\|\|)$ if there is a positive number $M$ such that

$$
\left\|\sum_{k=1}^{n} \alpha_{k} x_{k}\right\| \geqq M \sum_{k=1}^{n}\left|\alpha_{k}\right|
$$

for all $\alpha_{1}, \alpha_{2}, \cdots, \alpha_{n}$ in $\mathbf{C}$.

To see that the convolution operator $A$ is approximately finite dimenisonal, suppose $\epsilon>0$ is given. We use the fact that $\|g\| \leqq 1$ implies $f * g \in C(f)$

$$
C(f) \equiv \text { the uniform closure of }\left\{\sum_{k=1}^{p} \alpha_{k} T_{\zeta_{k}} f: p \in \mathbf{Z}^{+}, \sum_{k=1}^{p}\left|\alpha_{k}\right| \leqq 1\right\},
$$

where $T_{\zeta}$ is a translation operator; that is, $T_{\zeta} f(x) \equiv f(x+\zeta)(x \in \mathbf{R})$. It is not hard to show that $C(f)$ is totally bounded with respect to the uniform norm \|\|$_{\infty}[\mathbf{5}$, p. 77]. Hence, consider an $\epsilon$-approximation $g_{1}, g_{2}, \cdots, g_{m}$ to $C(f)$. It is shown in [5, p. 98] that either there is a linear independent subset of $\left\{g_{1}, g_{2}, \cdots, g_{m}\right\}$, call it $\left\{h_{1}, h_{2}, \cdots, h_{n}\right\}$, such that the distance from each $g_{j}$ to span $\left(h_{1}, h_{2}, \cdots, h_{n}\right)$ is small; that is, $d\left(g_{j}\right.$, $\left.\operatorname{span}\left(h_{1}, h_{2}, \cdots, h_{n}\right)\right)<\epsilon, \quad j=1,2, \cdots, m ;$ or $\left\|g_{j}\right\|<\epsilon$ for each $j, j=$ $1,2, \cdots, m$. In the latter case, the null operator is within $2 \epsilon$ of $A$. In the former case, the finite dimensional operator $P \circ A$, where $P$ is the projection onto $\operatorname{span}\left(h_{1}, h_{2}, \cdots, h_{n}\right)$, is within $2 \epsilon$ of $A$ because if $\|g\| \leqq 1$ we have $\left\|A g-g_{j}\right\| \leqq \epsilon$ for some $j$ and since $\left\|g_{j}-h^{\prime}\right\|<\epsilon$ for 
some $h \in \operatorname{span}\left(h_{1}, h_{2}, \cdots, h_{n}\right)$ we have that $\|P \circ A g-A g\| \leqq$ $\|h-A g\|<2 \epsilon$.

The constructive spectral properties of approximately finite dimensional operators are described in [5, pp. 16-49]. Applying these results to $A$ we can construct a sequence of subsets of the complex plane, $\left\{\sigma_{n}\right\}_{n=1}^{\infty}$, and a strictly decreasing sequence of positive numbers, $\left\{\rho_{n}\right\}_{n=1}^{\infty}$, such that $\rho_{n} \rightarrow 0$ and such that the following conditions are satisfied:

(i) For each positive integer $n$ either $\sigma_{n}$ is empty or $\sigma_{n}$ is the closure of some subset $\left\{\mu_{1}, \mu_{2}, \cdots, \mu_{q}\right\}$ of approximate eigenvalues of $A$.

(ii) $\sigma_{n} \subset\left\{z \in \mathbf{C}: \rho_{n+1}<|z|<\rho_{n}\right\}$ for eåch positive integer $n$.

(iii) If $\zeta \neq 0$ and the distance from $\zeta$ to each nonempty $\sigma_{n}$ is positive, then $(A-\zeta I)^{-1}$ exists.

REMARK. We do not claim that in case $\sigma_{n}$ is the closure of $\left\{\mu_{1}, \mu_{2}, \cdots, \mu_{q}\right\}$ that the $\mu_{k}$ 's are necessarily distinct or isolated from one another, since in constructive mathematics it is not true that two complex numbers are either distinct or equal.

REMARK. In general, we do not claim that the spectral points of an approximately finite dimensional operator are bona fide eigenvalues; that is, spectral points for which we can construct an eigenvector. However, an isolated spectral point of an approximately finite dimensional operator is a bona fide eigenvalue.

REMARK. This construction of the spectrum does not lead to a decision on whether or not zero is a spectral point. This is not surprising since if such a decision were always possible, then we could prove: $\alpha \in \mathbf{R}$ implies $\alpha=0$ or $\alpha \neq 0$. (To see this, consider the finite dimensional operator given by the matrix

$$
\left(\begin{array}{cc}
\alpha & 0 \\
0 & 1
\end{array}\right)
$$

Its determinant is $\alpha$. If the matrix is invertible then $\alpha \neq 0$; otherwise, $\alpha=0$.) Note that because the sets $\sigma_{n}$ near zero may or may not be empty, the spectrum is generally fuzzy near zero but quite clear away from zero. For a discussion of the spectral properties of approximately finite dimensional operators, see [5, pp. 16-49].

At this point, we construct finite dimensional, $A$-invariant subspaces by applying the functional calculus techniques developed for approximately finite dimensional operators in [5, pp. 50-66]. For each positive integer $n$, we get a projection operator $P_{\sigma_{n}}$ such that $E_{\sigma_{n}} \equiv\left\{P_{\sigma_{n}} h: h \in H\right\}$ is a finite dimensional, $A$-invariant subspace and such that the spectrum of the restriction of $A$ to $E_{\sigma_{n}}$ is $\sigma_{n}$ in case $\sigma_{n}$ is not empty. $\quad\left(P_{\sigma_{n}} \equiv 0\right.$ if $\sigma_{n}$ 
is empty.) Furthermore,

$$
A=\sum_{n=1}^{\infty} A \circ P_{\sigma_{n}}
$$

The proof of (1.2) follows from the constructive properties of the distribution of the spectral points of $A$ and the fact that $A$ is Hermitian. For example,

$$
\left\|A\left(I-\sum_{n=1}^{m} P_{\sigma_{n}}\right) h\right\| \leqq \rho_{m+1}\|h\|,
$$

since the spectrum of $A$ restricted to $\left(I-\sum_{n=1}^{m} P_{\sigma_{n}}\right) H$ is a subset of $\left\{z \in \mathbf{C}:|z|<\rho_{m+1}\right\}$ because of condition (ii) of the spectral properties of $A$.

We have now arrived at the most crucial stage of the construction of the trigonometric series for $f$. We will show that each $E_{\sigma_{n}}\left(\sigma_{n}\right.$ not empty) has a basis of exponential functions and the collection of these exponentials are the exponentials in the expansion of $f$. First we need a lemma.

Lemma. The spaces $E_{\sigma_{n}}\left(n \in \mathbf{Z}^{+}\right)$are invariant under all translation operators $T_{\zeta}(\zeta \in \mathbf{R})$.

The classical proof of this lemma fails to be valid from the constructive point of view because it ultimately relies on the assumption that the spectral points of the convolution operator $A$ are isolated from one another.

The constructive proof begins by showing that $A$ commutes with each translation operator $T_{\zeta}$. To see this consider for each $x \in \mathbf{R}$

$$
\begin{aligned}
A\left(T_{\zeta} g\right)(x) & =\underset{t}{M}\{f(x-t) g(t+\zeta)\} \\
& =\underset{t}{M}\{f(x+\zeta-t) g(t)\} \\
& =[A g](x+\zeta) \\
& =T_{\zeta}(A g)(x) .
\end{aligned}
$$

We claim that $E_{\{\mu\}}$ is invariant under all translations in case $\mu \neq 0$ is an isolated spectral point of $A$. To see this note that $A=\mu I$ on $E_{\{\mu\}}$ because the Hermitian operator $A-\mu I$ on $E_{\{\mu\}}$ has spectrum $\{0\}$, so $A-\mu I$ is bounded by each positive number; that is, $A-\mu I \equiv 0$ on $E_{\{\mu\}}$. Since $A-\mu I$ is invertible on the orthogonal complement of $E_{\{\mu\}}$ 
and since $(A-\mu I)\left(T_{\zeta} h-P_{\{\mu\}} T_{\zeta} h\right)=0$ because $A T_{\zeta} h=T_{\zeta} A h=\mu T_{\zeta} h$, it follows that $T_{\zeta} h-P_{\{u\}} T_{\zeta} h=0$ whenever $h \in E_{\{\mu\}}$; that is, $T_{\zeta} h \in E_{\{\mu\}}$ whenever $h \in E_{\{\mu\}}$.

Now consider any spectral set $\sigma$, say, and the space $E_{\sigma}$ where $\sigma$ is the closure of the set of spectral points $\left\{\mu_{1}, \mu_{2}, \cdots, \mu_{q}\right\}$. We will show that such spaces $E_{\sigma}$ are invariant under all translations by induction on $q$. We just showed that $E_{\sigma}$ is invariant under all translations in case $q=1$. Assume $E_{\sigma}$ is invariant under all translations whenever the spectral set $\sigma$ is the closure of a set of spectral points $\left\{\lambda_{1}, \lambda_{2}, \cdots, \lambda_{p}\right\}$ and $p<q$. To show that this implies $E_{\sigma}$ is invariant under all translations consider $\epsilon>0$. Compute a number $r$ such that $0<r<\epsilon$ and $r$ is in the metric complement of $\left\{0,\left|\mu_{1}-\mu_{2}\right|,\left|\mu_{1}-\mu_{3}\right|, \cdots,\left|\mu_{1}-\mu_{q}\right|\right\}$. Then for each $k, 1 \leqq k \leqq q$, either $\left|\mu_{1}-\mu_{q}\right|<r$ or $\left|\mu_{1}-\mu_{k}\right|>r$. Hence, either $\left|\mu_{1}-\mu_{k}\right|<r<\epsilon$ for all $k, 1 \leqq k \leqq q$, or $\sigma$ splits into two smaller spectral sets. In the latter case we can write $\sigma=\sigma_{1} \cup \sigma_{2}$ where $\sigma_{1}$ is the closure of $\left\{\mu_{i_{1}}, \mu_{t_{2}}, \cdots, \mu_{i_{1}}\right\}$ and $\sigma_{2}$ is the closure of $\left\{\mu_{i_{1+1}}, \mu_{i_{++2}}, \cdots, \mu_{i_{q}}\right\}\left(\mu_{i_{1}}, \cdots, \mu_{i_{q}}\right.$ is a permutation of $\mu_{1}, \mu_{2}, \cdots, \mu_{q}$ ); where $\sigma_{1}, \sigma_{2}$ are isolated from one another. So in the latter case we have the orthogonal decomposition $E_{\sigma}=E_{\sigma_{1}} \oplus E_{\sigma_{2}}$ (cf. [5, pp. 56-57]) and we can conclude that $E_{\sigma}$ is invariant under all translations since, by assumption, each of the $E_{\sigma_{t}}$ $(i=1,2)$ are. In the former case, in which $\left|\mu_{1}-\mu_{k}\right|<\epsilon$ for each $k$, $1 \leqq k \leqq q$, it can be shown that if $\zeta \in \mathbf{R}$ then we can compute a positive number $C$ independent of $\epsilon$ such that $\left\|T_{\zeta} h-P_{\sigma} T_{\zeta} h\right\| \leqq C \epsilon\|h\|$, whenever $h \in E_{\sigma}$. (See [5, p. 81].) Evidently, this inequality is valid in either case. Since $\epsilon$ was arbitrary it follows that $T_{\zeta} h \in E_{\sigma}$ whenever $h \in$ $E_{\sigma}$. This concludes the inductive proof of the lemma.

As shown in [5, p. 106-120], the fact that $E_{\sigma_{n}}$ is invariant under all translations whenever $\sigma_{n}$ is not empty implies that $E_{\sigma_{n}}$ has an orthonormal basis $\left\{h_{1}, h_{2}, \cdots, h_{N}\right\}$, say, such that each basis element is an eigenvector for each translation operator $T_{\zeta}$. We will show that this basis can be replaced by an orthonormal basis consisting of exponential functions.

We construct the new basis of exponentials by defining for each integer $j, 1 \leqq j \leqq N$, the function $e_{j}$, where $e_{j}(x) \equiv\left(T_{x} h_{l}, h_{l}\right)$; that is, $T_{x} h_{j}=e_{l}(x) h_{j}(x \in \mathbf{R})$. Note that each $e_{j}$ satisfies the following properties for all $x, y \in \mathbf{R}$ :

(i) $e_{j}(x+y)=e_{\jmath}(x) e_{j}(y)$.

(ii) $e_{j}$ is continuous.

(iii) $e_{j}(0)=1$ and $\left|e_{j}(x)\right|=1$.

(Property (ii) follows from the fact that $\left\|T_{x} h-T_{y} h\right\|$ is exceeded by $\left\|T_{x} h-T_{y} h\right\|_{\infty}$, which approaches zero as $|x-y|$ approaches zero, whenever $h$ is almost periodic.) It is evident from these properties that we can find real numbers $\eta_{1}, \eta_{2}, \cdots, \eta_{N}$ such that for each $j, 1 \leqq j \leqq N$, $e_{,}(x)=\exp \left\{i \eta_{,} x\right\}(x \in \mathbf{R})$. 
Now the fact that $\left\|T_{x} h_{j}-e^{i \eta_{j} x} h_{j}\right\|=0$ for each $x \in \mathbf{R}$ suggests that $h_{j}$ is exponential. In fact, it is not hard to show that $e_{j}=\left(e_{j}, h_{j}\right) h_{j}$ $(j=1,2, \cdots, N)$. Consequently, since $\left.\mid\left(e_{j}, h_{j}\right)\right\}=1$ for each $j$ $(j=1,2, \cdots, N), e_{1}, e_{2}, \cdots, e_{N}$ is an orthonormal basis of exponentials for $E_{\sigma_{n}}$.

To show that $e_{j}=\left(e_{j}, h_{j}\right) h_{j}$ for each $j$, first we note that $e_{j} \in H$ since $e_{j}(x)=\left(T_{x} h_{j}, h_{j}\right)=h_{j} * h_{j}^{*}(x)$ and $H$ is closed under convolution and involution. Now consider any vector $\varphi$ orthogonal to $h_{j}$. We have

$$
\begin{aligned}
\left(e_{j}, \varphi\right) & =\underset{x}{M}\left\{e_{j}(x) \bar{\varphi}(x)\right\} \\
& =\underset{x}{M}\left\{\left(T_{x} h_{j}, h_{j}\right) \bar{\varphi}(x)\right\} \\
& =\underset{x}{M}\left\{\underset{t}{M}\left\{h_{j}(x+t) \bar{h}_{j}(t)\right\} \bar{\varphi}(x)\right\} \\
& =\underset{t}{M}\left\{\underset{x}{M}\left\{T_{t} h_{j}(x) \bar{\varphi}(x)\right\} \overline{h_{j}}(t)\right\} \\
& =M_{t}\left\{e_{j}(t)\left(h_{j}, \varphi\right) \overline{h_{j}(t)}\right\},
\end{aligned}
$$

since $T_{t} h_{j}=e_{j}(t) h_{j} . \quad$ But $\left(h_{j}, \varphi\right)=0$, hence $\left(e_{j}, \varphi\right)=0$. We conclude that

$$
e_{j}=\left(e_{j}, h_{j}\right) h_{j} \quad(j=1,2, \cdots, N)
$$

and that $e_{1}, e_{2}, \cdots, e_{N}$ is an orthogonal basis for $E_{\sigma_{n}}$.

We have now reached the final stages of the construction of the expansion of $f$. The expansion we are about to give is somewhat less palatable than Bohr's.

We can collect all the exponentials produced by the bases of the spaces $E_{\sigma_{1}}, E_{\sigma_{2}}, \cdots$ in a sequence $\varphi_{1}, \varphi_{2}, \varphi_{3}, \cdots$, say. But since some of the $\sigma_{n}$ 's may be empty the following properties are satisfied for any pair of integers $j, k$ :

(i) $\left\|\varphi_{j}\right\|=0$ or $\left\|\varphi_{j}\right\|=1$.

(ii) $\left\|\varphi_{j}\right\|=1$ implies $\varphi_{j}(x) \equiv e^{i \lambda_{j} x}$ for some $\lambda_{j} \in \mathbf{R}$.

(iii) $\left(\varphi_{j}, \varphi_{k}\right)=0$ whenever $j \neq k$.

In order to show that $f \sim \sum_{k=1}^{\infty}\left(f, \varphi_{k}\right) \varphi_{k}$, recall from 1.2 that

$$
A=\sum_{n=1}^{\infty} A \circ P_{\sigma_{n}} .
$$

We can write this last equation in the form

$$
f * g=\sum_{k=1}^{\infty}\left(g, \varphi_{k}\right) f * \varphi_{k} . \quad(g \in H)
$$


But for each positive integer $k, f * \varphi_{k}=\left(f, \varphi_{k}\right) \varphi_{k}$ since $\varphi_{k}$ is either the zero function or an exponential. Thus 1.5 becomes

$$
f * g=\sum_{k=1}^{\infty}\left(g, \varphi_{k}\right)\left(f, \varphi_{k}\right) \varphi_{k} . \quad(g \in H) .
$$

Note that the right hand side of 1.6 converges uniformly since

$$
\begin{aligned}
\left|\sum_{k=n}^{m}\left(f, \varphi_{k}\right)\left(g, \varphi_{k}\right) \varphi_{k}(x)\right| & =\left|f *\left(\sum_{k=n}^{m}\left(g, \varphi_{k}\right) \varphi_{k}(x)\right)\right| \\
& \leqq\|f\|\left\|\sum_{k=n}^{m}\left(g, \varphi_{k}\right) \varphi_{k}\right\| \\
& =\|f\|\left[\sum_{k=n}^{m}\left|\left(g, \varphi_{k}\right)\right|^{2}\right]^{1 / 2} .
\end{aligned}
$$

Thus we can write

$$
\|f\|^{2}=f * f(0)=\sum_{k=1}^{\infty}\left(f, \varphi_{k}\right)\left(f, \varphi_{k}\right) \varphi_{k}(0) .
$$

But $\varphi_{k}(0)=0$ if $\varphi_{k} \equiv 0$ and $\varphi_{k}(0)=1$ if $\varphi_{k}$ is an exponential; hence, $\left(f, \varphi_{k}\right)\left(f, \varphi_{k}\right) \varphi_{k}(0)=\left(f, \varphi_{k}\right)^{2}$. Since $\left(f, \varphi_{k}\right)$ is real for each $k, 1.7$ becomes

$$
\|f\|^{2}=\sum_{k=1}^{\infty}\left|\left(f, \varphi_{k}\right)\right|^{2}
$$

This equation together with the fact that

$$
\left\|f-\sum_{k=1}^{m}\left(f, \varphi_{k}\right) \varphi_{k}\right\|=\|f\|^{2}-\sum_{k=1}^{m}\left|\left(f, \varphi_{k}\right)\right|^{2}
$$

establishes the fundamental result

$$
f=\sum_{k=1}^{\infty}\left(f, \varphi_{k}\right) \varphi_{k}
$$

where convergence is in the \|\| -sense.

2. Uniform approximation theorem. The existence of the trigonometric expansion of a continuous almost periodic function is the basis of each of the classical proofs for the uniform approximation theorem, which states that each continuous almost periodic function is the uniform limit of a sequence of trigonometric polynomials - Bohr's 
second result. Bohr [4] employed the theory of purely periodic functions of infinitely many variables to obtain a classical proof of this result. Bochner [3] obtained a nonconstructive proof by extending the Fejér summation method to the class of continuous almost periodic functions. From the constructive point of view, we like a method due to Weyl (cf. [1, pp. 29-31]) which, with a few easy modifications and with the computation of the trigonometric expansion now established, can be adopted to furnish a constructive proof.

Approximation Theorem. Suppose $f$ is a continuous almost periodic function with expansion

$$
f=\sum_{k=1}^{\infty}\left(f, \varphi_{k}\right) \varphi_{k}
$$

Then for each $\epsilon>0$ there is a trigonometric polynomial $P(x)$ of the form

$$
P(x)=\sum_{j=1}^{p} c_{j} e^{i \lambda_{j} x}, \quad(x \in \mathbf{R})
$$

where the frequencies $\lambda_{1}, \lambda_{2}, \cdots, \lambda_{p}$ are among those frequencies of $f$, and such that

$$
|f(x)-P(x)|<\epsilon . \quad(x \in \mathbf{R}) .
$$

Given $\epsilon>0$, we begin the construction of $P$ by considering, for each positive integer $m$,

$$
f_{m} \equiv f-\sum_{j=1}^{m}\left(f, \varphi_{j}\right) \varphi_{j}
$$

By the Parseval equation we have

$$
\underset{x}{M}\left\{\left|f_{m}(x)\right|^{2}\right\}=\sum_{j=m+1}^{\infty}\left|\left(f, \varphi_{j}\right)\right|^{2}
$$

Given any $\eta>0$, we can find an $m$ such that

$$
\underset{x}{M}\left\{\left|f_{m}(x)\right|^{2}\right\}<\eta
$$

since $\sum_{j=1}^{\infty}\left|\left(f, \varphi_{j}\right)\right|^{2}$ is a convergent series.

The crucial step in Weyl's proof is the application of the following lemma, which is proven in $[\mathbf{1}, \mathrm{p} .15]$. 
LEMma. There exists $T_{0}>0$ such that

$$
\left.\left|\frac{1}{T} \int_{0}^{T}\right| f_{m}(x+s)\right|^{2} d x-\underset{x}{M}\left\{\left|f_{m}(x+s)\right|^{2}\right\} \mid<\eta
$$

for all $T>T_{0}$ and $s \in \mathbf{R}$.

It follows from 2.3 and 2.2 that

$$
\frac{1}{T} \int_{0}^{T}\left|f_{m}(x+s)\right|^{2} d x<2 \eta
$$

for all $s \in \mathbf{R}$ and $T>T_{0}$.

Now let $L$ be a modulus of almost periodicity for $f$. Find a positive integer $N$ such that $T=N(L(\epsilon / 3)+1)>T_{0}$. In each of the intervals $[k(L(\epsilon / 3)+1), k(L(\epsilon / 3)+1)+L(\epsilon / 3)], k=0,1,2, \cdots, N-1$; select an $\epsilon / 3$-translation number $t_{k}$, say.

Let $\omega$ be a modulus of uniform continuity for $f$ such that $0<$ $\omega(\epsilon / 3)<1$.

We define a function $\chi$ on a dense subset of $[0, T]$ such that $\chi(x) \equiv 1$ on the intervals $\left[t_{k}, t_{k}+\omega(\epsilon / 3)\right]$ and $\chi(x) \equiv 0$ on the metric complement (in $[0, T]$ ) of their union. (Note that the $\left[t_{k}, t_{k}+\omega(\epsilon / 3)\right]$ do not overlap.)

Because of the Schwarz inequality, we have

$$
\left|\int_{0}^{T} f_{m}(x+s) \chi(x) d x\right|^{2} \leqq \int_{0}^{T}\left|f_{m}(x+s)\right|^{2} d x \int_{0}^{T} \chi(x)^{2} d x
$$

for all $s \in \mathbf{R}$. Also, note that

$$
\begin{aligned}
\int_{0}^{T} f_{m}(x+s) \chi(x) d x & =\sum_{k=0}^{N-1} \int_{t_{k}}^{t_{k}+\omega(\epsilon / 3)} f_{m}(x+s) d x \\
& =\sum_{k=0}^{N-1} \int_{0}^{\omega(\epsilon / 3)} f_{m}\left(x+t_{k}+s\right) d x
\end{aligned}
$$

for all $s \in \mathbf{R}$, and that

$$
\int_{0}^{T} \chi^{2}(x) d x=N \omega(\epsilon / 3) .
$$

Now it follows from $2.4,2.5,2.6$ and 2.7 that

$$
\left|\sum_{k=0}^{N-1} \int_{0}^{\omega(\epsilon / 3)} f_{m}\left(x+t_{k}+s\right) d x\right|<\sqrt{2 \eta T N \omega(\epsilon / 3)}
$$


for all $s \in \mathbf{R}$. Because $T=N(L(\epsilon / 3)+1)$, the inequality in 2.8 can be restated in the form

$$
\left|\frac{1}{N \omega(\epsilon / 3)} \sum_{k=0}^{N-1} \int_{0}^{\omega(\epsilon / 3)} f_{m}\left(x+t_{k}+s\right) d x\right|<\sqrt{\frac{2 \eta(L(\epsilon / 3)+1)}{\omega(\epsilon / 3)}}
$$

for all $s \in \mathbf{R}$.

Now suppose we had chosen $\eta$ such that

$$
\eta<\frac{\epsilon^{2} \omega(\epsilon / 3)}{18(L(\epsilon / 3)+1)}
$$

then 2.9 becomes

$$
\left|\frac{1}{N \omega(\epsilon / 3)} \sum_{k=0}^{N-1} \int_{0}^{\omega(\epsilon / 3)} f_{m}\left(x+t_{k}+s\right) d x\right|<\epsilon / 3
$$

for all $s \in \mathbf{R}$.

At this point we want to express $f_{m}$ in terms of exponential functions. Ignoring the cases $\varphi_{j} \equiv 0(j=1,2, \cdots, m)$, we can write 2.1 in the form

$$
f(x)-\sum_{j=1}^{p} \alpha_{\jmath} e^{i \lambda_{1} x}=f_{m}(x)
$$

where $e^{i \lambda_{j} x}$ are exponentials in the expansion of $f$ and $\alpha_{l} \equiv\left(f(x), e^{i \lambda_{j} x}\right)$.

Now it follows from 2.11 that for each $k(k=0,1, \cdots, N-1)$ we have

$$
\frac{1}{N \omega(\epsilon / 3)} \int_{0}^{\omega(\epsilon / 3)} f_{m}\left(x+t_{k}+s\right) d x
$$

$$
=\frac{1}{N \omega(\epsilon / 3)} \int_{0}^{\omega(\epsilon / 3)} f\left(x+t_{k}+s\right)-P_{k}(s)
$$

where

$$
P_{k}(s) \equiv \frac{1}{N \omega(\epsilon / 3)} \sum_{j=1}^{p} \alpha_{\jmath} e^{i \lambda_{j} s} \int_{0}^{\omega(\epsilon / 3)} e^{i \lambda_{l}\left(x+t_{k}\right)} d x
$$

for $s \in \mathbf{R}$. Note that $P_{k}(k=0,1, \cdots, N-1)$ is a trigonometric polynomial with exponentials belonging to the expansion of $f$.

As a consequence of 2.12 , we can write the inequality in 2.10 in the form 


$$
\left|\frac{1}{N \omega(\epsilon / 3)} \sum_{k=0}^{N-1} \int_{0}^{\omega(\epsilon / 3)} f\left(x+t_{k}+s\right) d x-P(s)\right|<\epsilon / 3
$$

for all $s \in \mathbf{R}$, where

$$
P(s) \equiv \sum_{k=0}^{N-1} P_{k}(s)
$$

Note that $P(s)$ is a trigonometric polynomial with exponentials among those in the expansion of $f$.

Now we can use the fact that $\left|f\left(x+t_{k}+s\right)-f\left(t_{k}+s\right)\right|<\epsilon / 3$ for each $k$ and for each $x$ such that $0 \leqq x \leqq \omega(\epsilon / 3)$ to write

$$
\left|\frac{1}{\omega(\epsilon / 3)} \int_{0}^{\omega(\epsilon / 3)} f\left(x+t_{k}+s\right) d x-f\left(t_{k}+s\right)\right|<\epsilon / 3
$$

for each $k(k=0,1, \cdots, N-1)$ and all $s \in \mathbf{R}$.

Next, note that $\left|f\left(t_{k}+s\right)-f(s)\right|<\epsilon / 3$ for all $s \in \mathbf{R}$ since each $t_{k}$ is an $\epsilon / 3$-translation number. This fact together with 2.14 establishes the inequality

$$
\left|\frac{1}{\omega(\epsilon / 3)} \int_{0}^{\omega(\epsilon / 3)} f\left(x+t_{k}+s\right)-f(s)\right|<2 \epsilon / 3
$$

for each $k(k=0,1, \cdots, N-1)$ and all $s \in \mathbf{R}$.

Finally, we combine inequality 2.15 with 2.13 to get $|f(s)-P(s)|<\epsilon$ for all $s \in \mathbf{R}$.

\section{REFERENCES}

1. A. Besicovitch, Almost Periodic Functions, Dover Publications, Inc. (1954).

2. E. Bishop, Foundations of Constructive Analysis, McGraw Hill Book Company (1967).

3. S. Bochner, Beiträge zur Theorie der fastperiodischen Funktionen, I, II, Math. Annalen, 96 (1926), 119-147, 383.

4. H. Bohr, Zur Theorie der fastperiodischen Funcktionen, I, II, III, Acta Math., 45 (1924), 29-127.

5. J. D. Brom, A constructive version of the fundamental theorem of the theory of almost periodic functions, Thesis, University of California, San Diego, 1974.

6. C. G. Gibson, On the almost periodicity of trigonometric polynomials in constructive mathematics, Nederl. Akad. Wetensch. Proc. Ser. A75 = Inadag. Math., 34 (1972), 355-361.

7. F. Rellich, Über die v. Neumannschen fast periodischen funktionen auf einer Gruppe, Math. Annalen, III (1935), 560-567.

8. H. Weyl, Integralgleichungen and fastperiodische Funktionen, Math. Annalen, 97 (1927), 338-356.

Received October 13, 1976.

UNIVERSITY OF CALIFORNIA

SANTA BARbara, CA 93106

Current address: RockHURST COLLEgE

Kansas City, MO 64110 




\section{Pacific Journal of Mathematics \\ Vol. 70, No. $1 \quad$ September, 1977}

William H. Barker, Noether's theorem for plane domains with hyperelliptic

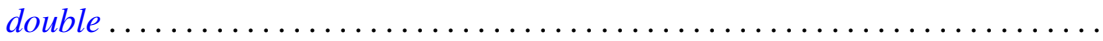

Michael James Beeson, Non-continuous dependence of surfaces of least area on the boundary curve ...................................... 11

Horst Behncke, Functions acting in weighted Orlicz algebras . . . . . . . . . . . . 19

Howard Edwin Bell, A commutativity study for periodic rings . . . . . . . . . . . 29

Peter Botta and Stephen J. Pierce, The preservers of any orthogonal group ....... 37

Douglas S. Bridges, The constructive Radon-Nikodým theorem ............. 51

James Dennis Brom, The theory of almost periodic functions in constructive

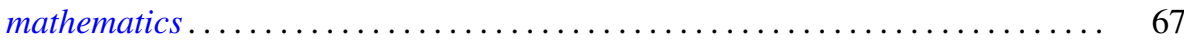

N. Burgoyne and C. Williamson, Semi-simple classes in Chevalley type groups ....

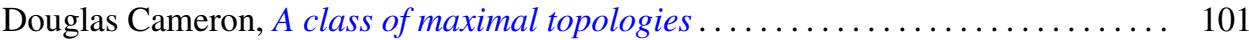

L. Carlitz, Enumeration of doubly up-down permutations . . . . . . . . . . . . . . 105

Paul Robert Chernoff, The quantum n-body problem and a theorem of

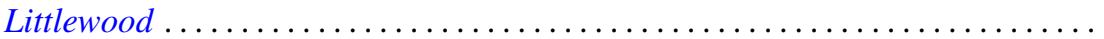

Jo-Ann Deborah Cohen, Locally bounded topologies on $F(X) \ldots \ldots \ldots \ldots \ldots \ldots$

Heinz Otto Cordes and Robert Colman McOwen, Remarks on singular elliptic

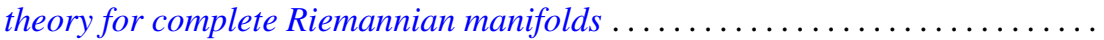

Micheal Neal Dyer, Correction to: "Rational homology and Whitehead

products"

Robert Fernholz, Factorization of Radonifying transformations

Lawrence Arthur Fialkow, A note on quasisimilarity. II ...... . .

Harvey Charles Greenwald, Lipschitz spaces of distributions on the surface of unit

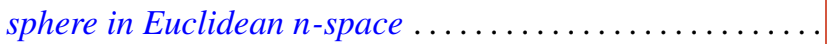

Albrecht Irle, On the measurability of conditional expectations

Tom (Roy Thomas Jr.) Jacob, Matrix transformations involving simple sequence

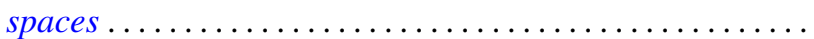

A. Katsaras, Continuous linear maps positive on increasing continuous

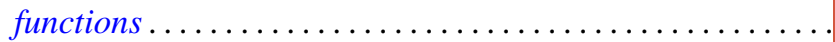

Kenneth Kunen and Judith Roitman, Attaining the spread at cardinals of cofinality

Lawrence Louis Larmore and Robert David Rigdon, Enumerating normal bundles

of immersions and embeddings of projective spaces ...... . .

Ch. G. Philos and V. A. Staïkos, Asymptotic properties of nonoscillatory solutions of differential equations with deviating argument .

Peter Michael Rosenthal and Ahmed Ramzy Sourour, On operator algebras containing cyclic Boolean algebras...

Polychronis Strantzalos, Strikt fast gleichgradig-stetige und eigentliche

Aktionen ...

Glenn Francis Webb, Exponential representation of solutions to an abstract

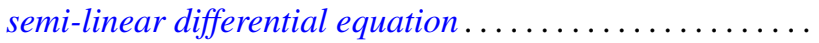

